ARTÍCULO DE INVESTIGACIÓN

\title{
Estimación de contenido de carbono en la cuenca del río Magdalena, México
}

\author{
Estimation of carbon content \\ in the Magdalena River basin, Mexico
}

\author{
José Mauricio Galeana-Pizaña ${ }^{1}$, \\ José Antonio Benjamín Ordóñez-Díaz ${ }^{2}$ y Nirani Corona-Romero ${ }^{1}$
}

\begin{abstract}
RESUMEN
En el presente estudio se estimó el contenido de carbono de la biomasa aérea de seis tipos de cobertura vegetal en la cuenca del río Magdalena, ubicada en el suelo de conservación del Distrito Federal. Para ello, se elaboró un mapa de las clases de cobertura vegetal y uso de suelo del área de estudio, mediante la fotointerpretación de fotografías aéreas. Se utilizó un diseño de muestreo jerárquico estratificado con distribución sistemática, los sitios resultantes se desplegaron en un enmallado con una equidistancia de 550 metros. La colecta de los datos en campo se basó en un diseño anidado; con una intensidad de muestreo de $0,64 \%$. Se establecieron 52 sitios de muestreo de $3000 \mathrm{~m}^{2}$ cada uno. La cobertura vegetal que presentó mayor cantidad de carbono almacenado corresponde a la clase de Abies religiosa cerrado, con $163 \mathrm{Mg} \mathrm{C}^{-1}$ promedio; le sigue la clase de Abies religiosa - Pinus hartwegii cerrado con $107 \mathrm{Mg} \mathrm{C}^{-1}$ y la clase de Abies religiosa abierto con $95 \mathrm{Mg} \mathrm{C} \mathrm{ha-1}^{-1}$. Las clases de Pinus sp. y Pinus hartwegii cerrado tuvieron $47 \mathrm{Mg} \mathrm{C}^{-1}$; Pinus hartwegii - Abies religiosa cerrado, $39 \mathrm{Mg} \mathrm{C}$ ha $^{-1}$ y Pinus hartwegii abierto, $21 \mathrm{Mg} \mathrm{C} \mathrm{ha}^{-1}$.
\end{abstract}

PALABRAS CLAVE:

Abies religiosa, biomasa aérea, bosques, estructura diamétrica, Pinus hartwegii.

\begin{abstract}
Carbon content of aboveground biomass of six types of vegetation cover in the Magdalena River basin, located in the conservation land in the Mexico City, was estimated. Hence, a map of cover vegetation and land use to the study area was made; a hierarchical stratified sampling design with distribution systematic with a mesh of sampling sites equidistant of 550 meters was used for field work. The method used for field data collection was based on a nested design, with an intensity of sample of $0,64 \%$. There were generated 52 sites of $3000 \mathrm{~m}^{2}$. The vegetation cover that showed the highest amount of carbon stored corresponds to the forest of the the Abies religiosa closed with $163 \mathrm{Mg} \mathrm{C} \mathrm{ha}^{-1}$ in average; followed by the Abies religiosa - Pinus hartwegii closed with $107 \mathrm{Mg} \mathrm{C} \mathrm{ha-}^{-1}$, and Abies religiosa opened with $95 \mathrm{Mg} \mathrm{C}^{-1}$; about the covers of Pinus sp., Pinus hartwegii closed with $47 \mathrm{Mg} \mathrm{C}^{-1}$; Pinus hartwegii - Abies religiosa closed with $39 \mathrm{Mg} \mathrm{C} \mathrm{ha-1}^{-1}$ and Pinus hartwegii opened with $21 \mathrm{Mg} \mathrm{C} \mathrm{ha-1}^{-1}$.
\end{abstract}

KEY WORDS:

Abies religiosa, above ground biomass, forest, diametrical structure, Pinus hartwegii. 


\section{INTRODUCCIÓN}

El cambio climático se define como las modificaciones termodinámicas que ocurren en la atmósfera a escala global, como consecuencia del incremento de las concentraciones de gases de efecto invernadero (GEI), provocando el calentamiento del planeta (IPCC, 2007). Esto afecta directamente al hombre y a las actividades económicas que tienen una correlación con el estado del tiempo (e.g.: temperatura, presión atmosférica, precipitación, etc.) produciendo modificaciones en su medio (e.g. aumento del nivel de mar), lo que a largo plazo provocará una crisis social (Galeana, 2008).

En el año 1976, se reconoció a los bosques como uno de los principales sumideros de carbono, junto con los océanos, el suelo y los pastizales (Mena, 2004). Actualmente, los ecosistemas boscosos están siendo amenazados por el cambio de uso de suelo, la deforestación y la quema de combustibles fósiles, siendo éstas las principales causas a nivel mundial de la liberación de dióxido de carbono $\left(\mathrm{CO}_{2}\right)$ a la atmósfera (Schneider, 1989; Goudie, 1990; Dixon et al., 1994). Los cambios en el uso del suelo y cobertura vegetal asociados a la fragmentación de hábitats son los disturbios más severos de las actividades antrópicas, causando un aumento de las tasas de extinción de especies, sobre todo en las décadas recientes (Soulé y Orians, 2001). Lo anterior indica un severo problema que se está acentuando y que se relaciona directamente con el cambio en la cobertura vegetal, lo cual repercute en el deterioro del ambiente (Lambin, 1994; Ordóñez, 2008).

La deforestación genera, a nivel regional, pérdida de la cubierta vegetal, detrimento de los recursos forestales, deterioro físico y químico del suelo, alteración del balance hídrico y desestabilización de cuencas; a nivel global, afecta el albedo y el balance de agua atmosférica, perturbando los patrones climáticos, contribuyendo así al calentamiento global (Houghton, 1991; Johns et al., 1992; Lund, 2006). Otra consecuencia es la reducción y fragmentación del hábitat (Murcia, 1995) lo que se traduce en pérdida de la biodiversidad y eliminación de la variabilidad genética de poblaciones y especies (Johns et al., 1992), sumado a una pérdida de los servicios ambientales y bioculturales (Otegui, 2007). Es por ello que estudios relacionados a los cambios en la cobertura vegetal están teniendo mayor atención (Brown y Lugo, 1994).

Históricamente, en una escala global, los cambios en la cobertura forestal han sido portadores de $\mathrm{CO}_{2}$ a la atmósfera (Houghton, 1991; Brown, 2001), por esta razón en la actualidad se considera a los bosques como una opción para mitigar las emisiones de GEI, ya que secuestran y mantienen más $\mathrm{CO}_{2}$ que otros ecosistemas terrestres, aparte de participar con $90 \%$ del flujo anual de carbono en el sistema atmósfera-tierra (Masera, 1995). EI IPCC (2007) estima que combinando estrategias de conservación forestal con proyectos de reforestación en el mundo, los bosques podrían resultar en un sumidero neto de carbono durante los próximos 100 años, permitiendo reducir de $20 \%$ a $50 \%$ las emisiones de $\mathrm{CO}_{2}$ a la atmósfera.

Las opciones de mitigación de los GEI pueden ser agrupadas en tres: La primera categoría incluye a las actividades que evitan la liberación del carbono secuestrado (e.g.: conservación y protección a los ecosistemas forestales); la segunda contiene actividades que capturan $\mathrm{CO}_{2}$ (e.g.: aforestación, reforestación y agroforestería); y la tercera opción encierra actividades que sustituyan el uso de combustibles fósiles (Sathaye et al., 2001). Los procesos de captura-emisión son parte de un sistema con cuatro tipos generales de reservorios de carbono (vegetación aérea y subterrá- 
nea, materia en descomposición, suelos y productos forestales), con tiempos de residencia y flujos asociados muy diferentes (Ordóñez, 1999).

Actualmente es creciente la atención global sobre el estimar con mayor certeza y precisión la dinámica del carbono secuestrado y emitido (balance emisióncaptura) entre el bosque y la atmósfera. Reconocer el papel que juegan los bosques en el ciclo global del carbono, particularmente en la mitigación de los GEI, es una prioridad (Brown, 2001). La estimación de la captura de carbono es el punto de partida para hacer efectivas las opciones de mitigación de los GEI en el marco del Protocolo de Kyoto, además de permitir establecer un criterio de base de cálculo de la dinámica de este gas cuando no se ejecuta una medida de mitigación (Masera et al., 2000); es decir, generar un caso de referencia con el cual se pueda comparar el cambio en las emisiones de IOS GEI y verificar los beneficios adicionales posteriores (IPCC, 2007).

La cuenca del río Magdalena brinda servicios ambientales a la ciudad de México, como son captura de carbono, recarga de mantos acuíferos, producción de oxígeno, fijación del sustrato y belleza escénica, principalmente; lo anterior sugiere acciones inmediatas dirigidas a la conservación y adecuado manejo de sus recursos. Sin embargo, la zona ha recibido fuertes presiones antropogénicas como asentamientos humanos, procesos de cambio de uso de suelo, instalaciones de infraestructura para diversos fines, extracción de recursos naturales (madera y fauna nativa, principalmente) y turismo no regulado, lo que ha ocasionado, entre otros problemas: deforestación y degradación forestal, establecimiento y auge de plagas forestales, incendios, contaminación del acuífero, inseguridad y erosión. Por ello, es primordial el desarrollo de una línea base confiable para iniciar proyectos de captura de carbono en los bosques del río Magdalena, lo que permita entender el proceso del cambio en la cobertura vegetal y uso del suelo y así poder ofrecer alternativas de mitigación del cambio climático (Fernández et al., 2002; Galeana, 2008).

\section{OBJETIVOS}

Los objetivos de la presente investigación son ,estimar el contenido de carbono en la biomasa aérea de las coníferas de la cuenca del río Magdalena, Distrito Federal, México; elaborar un mapa de cobertura vegetal y uso del suelo del área de estudio y, cuantificar el contenido de carbono por tipo de cobertura vegetal.

\section{METODOLOGÍA}

\section{Área de estudio}

La cuenca del río Magdalena se localiza dentro del suelo de conservación de las delegaciones Magdalena Contreras, Álvaro Obregón y Cuajimalpa, en el Distrito Federal (zona centro de México) (Fig. 1).

El área de estudio se encuentra ubicada en el suroeste de la cuenca de México, sobre la Sierra de las Cruces, misma que pertenece a la región fisiográfica del Sistema Volcánico Transversal, dentro de las subprovincia de Lagos y Volcanes de Anáhuac (INEGI, 2005a,b,c,d). Sus coordenadas extremas son: al Norte $19^{\circ} 17^{\prime} 52^{\prime \prime}$, al Sur $19^{\circ} 14^{\prime} 35^{\prime \prime}$, al Oeste $99^{\circ} 20^{\prime} 30^{\prime \prime}$ y al Este 99०15'06"' (GDF, 2000).

La cuenca tiene una superficie de 2981,3 ha de relieve accidentado (2480 msnm-3860 msnm) con bosques de Abies religiosa, Pinus hartwegii, Quercus spp. y mixto; sus climas son templado subhúmedo y semifrío subhúmedo, ambos con lluvias en verano y suelos de tipo andosol húmico (GDF, 2000). 

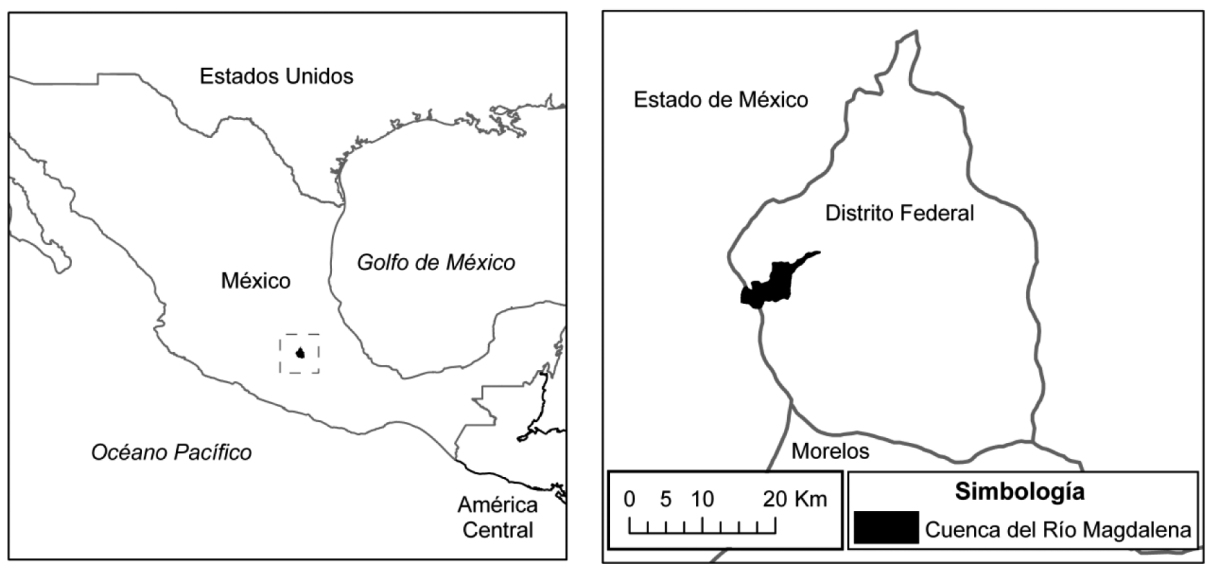

Figura 1. Localización de la cuenca del río Magdalena.

En la cuenca se forma el río Magdalena, que es uno de los cuerpos de agua más importantes de la Ciudad de México, con un volumen aproximado de agua permanente de $1 \mathrm{~m}^{3} \mathrm{~s}^{-1}$ y que es empleado como fuente de abastecimiento de agua potable (CNA, 1997).

\section{Elaboración del mapa de vegetación y uso de suelo}

Para realizar la estimación de captura de carbono se determinaron la clases de cobertura vegetal y uso de suelo para la elaboración del mapa, las cuales se definieron con base en los criterios de Reporte Técnico del Inventario Nacional Forestal (INF) de la Subsecretaría Forestal y Fauna Silvestre (SFFS, 1994), identificando las mismas categorías de cobertura vegetal y uso de suelo. Para la elaboración del mapa se reagruparon las clases de cobertura vegetal en 26 (Tabla 1).

Una vez determinadas las clases de cobertura vegetal, se realizó la interpreta- ción de fotografías aéreas de escala 1:10 000, tomadas en enero del año 2005 con dirección norte-sur, y proporcionadas por el Instituto de Geografía de la Universidad Nacional Autónoma de México. La digitación del mapa se generó en pantalla usando el software Autocad Map 2002 y la base de datos en ArcView 3.2a.

\section{Diseño de muestreo}

Se establecieron ocho clases de vegetación a muestrear para los fines de este estudio (Tabla 2).

Para estimar el contenido de carbono se utilizó un diseño de muestreo sistemático simple. Esto se hizo sobreponiendo una red cuadrangular de puntos equidistantes a 550 m (Fig. 2) a la capa de vegetación y uso de suelo, con el programa Arc GIS 9.2. El tamaño de muestra se determinó con $1 \%$ de intensidad de muestreo. Los puntos estuvieron definidos por coordenadas Universal Transversal de Mercator (UTM), representando el centro de las unidades a muestrear. 
Tabla 1. Relación de las clases de cobertura vegetal con las establecidas por el INF, 1994.

\begin{tabular}{|c|c|c|}
\hline Clases en este estudio & Claves & Clases del INF \\
\hline Agricultura & AGR & $\begin{array}{l}\text { Agricultura de riego } \\
\text { Agricultura de temporal }\end{array}$ \\
\hline Asentamientos humanos & AS & Zona urbana \\
\hline Bosque de Abies religiosa abierto & $\mathrm{BA}_{1}$ & Bosque de oyamel abierto \\
\hline Bosque de Abies religiosa cerrado & $\mathrm{BA}_{2}$ & Bosque de oyamel cerrado \\
\hline Bosque de Abies religiosa-Pinus hartwegii abierto & $\mathrm{BA}-\mathrm{Ph}_{1}$ & Bosques de otras coníferas abierto \\
\hline Bosque de Abies religiosa-Pinus hartwegii cerrado & $\mathrm{BA}-\mathrm{Ph}_{2}$ & Bosques de otras coníferas cerrado \\
\hline Bosque de Abies religiosa-Pinus sp. abierto & $\mathrm{BA}-\mathrm{Psp}_{1}$ & Bosques de otras coníferas abierto \\
\hline Bosque de Abies religiosa-Pinus sp. cerrado & BA-Psp & Bosques de otras coníferas cerrado \\
\hline Bosque de Abies religiosa-Quercus sp. abierto & $B A-Q_{1}$ & Bosques de otras coníferas abierto \\
\hline Bosque de Abies religiosa-Quercus sp. cerrado & $B A-Q_{2}$ & Bosques de otras coníferas cerrado \\
\hline Bosque de Quercus sp. abierto & $\mathrm{BQ}_{1}$ & Bosque de encino abierto \\
\hline Bosque de Quercus sp. cerrado & $\mathrm{BQ}_{2}$ & Bosque de encino cerrado \\
\hline Bosque de Quercus sp.-Abies religiosa abierto & $\mathrm{BQ}-\mathrm{A}_{1}$ & Bosques de otras coníferas abierto \\
\hline Bosque de Quercus sp.-Abies religiosa cerrado & $B Q-A_{2}$ & Bosques de otras coníferas cerrado \\
\hline Bosque de Pinus hartwegii abierto & $\mathrm{BPh}_{1}{ }^{2}$ & Bosque de pino abierto \\
\hline Bosque de Pinus hartwegii cerrado & $\mathrm{BPh}_{2}$ & Bosque de pino cerrado \\
\hline Bosque de Pinus sp. abierto & $\mathrm{BPsp}_{1}$ & Bosque de pino abierto \\
\hline Bosque de Pinus sp. cerrado & $\mathrm{BPsp}_{2}$ & Bosque de pino cerrado \\
\hline Bosque de Pinus hartwegii -Abies religiosa abierto & $\mathrm{BPh}-\mathrm{A}_{1}$ & Bosques de otras coníferas abierto \\
\hline Bosque de Pinus hartwegii -Abies religiosa cerrado & $\mathrm{BPh}-\mathrm{A}_{2}$ & Bosques de otras coníferas cerrado \\
\hline $\begin{array}{l}\text { Bosque de Pinus sp.- Abies religiosa abierto } \\
\text { Bosques de otras coníferas abierto }\end{array}$ & BPsp- $A_{1}$ & Bosques de otras coníferas abierto \\
\hline Bosque de Pinus sp.- Abies religiosa cerrado & BPsp- $A_{2}$ & Bosques de otras coníferas cerrado \\
\hline Bosque mixto & BM & No considerado \\
\hline Cuerpos de agua & $\mathrm{H}_{2} \mathrm{O}$ & Cuerpos de agua \\
\hline Pastizal & PAS & Pastizal inducido \\
\hline Plantación forestal & REF & Plantación forestal \\
\hline
\end{tabular}

Tabla 2. Clases de cobertura vegetal utilizadas para la estimación de carbono.

\begin{tabular}{|c|c|c|}
\hline Clases a muestrear & Claves & Clases del INF \\
\hline Bosque de Abies religiosa abierto & $\mathrm{BA}_{1}$ & Bosque de oyamel abierto \\
\hline Bosque de Abies religiosa cerrado & $\mathrm{BA}_{2}$ & Bosque de oyamel cerrado \\
\hline Bosque de Abies religiosa-Pinus hartwegii cerrado & $\mathrm{BA}-\mathrm{Ph}_{2}$ & $\begin{array}{l}\text { Bosques de otras coníferas } \\
\text { cerrado }\end{array}$ \\
\hline Bosque de Pinus hartwegii abierto & $\mathrm{BPh}_{1}$ & Bosque de pino abierto \\
\hline Bosque de Pinus hartwegii cerrado & $\mathrm{BPh}_{2}$ & Bosque de pino cerrado \\
\hline Bosque de Pinus hartwegii-Abies religiosa cerrado & $\mathrm{BPh}-\mathrm{A}_{2}$ & No considerado \\
\hline
\end{tabular}




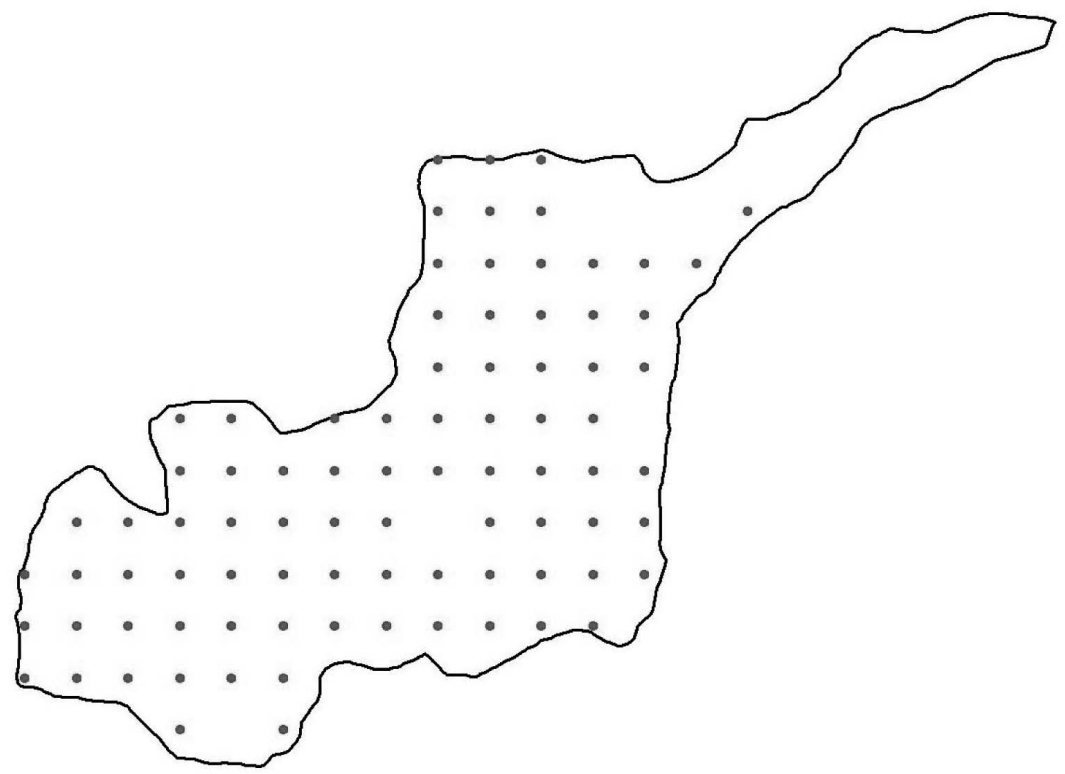

Figura 2. Red cuadrangular de puntos, obtenida del muestreo sistemático simple para la zona.

\section{Unidad de muestreo}

La metodología empleada en campo para la colecta de los datos de biomasa aérea se basó en un diseño anidado (Ordóñez, 2008; Fig. 3), que permitió la colecta de muestras y sus réplicas. De acuerdo con una intensidad de muestreo de $0,64 \%$, se generaron 52 sitios de $3000 \mathrm{~m}^{2}$, los cuales estuvieron conformados por tres parcelas circulares de $1000 \mathrm{~m}^{2}$ con un radio de $17,84 \mathrm{~m}$.

\section{Estimación del contenido de carbono en biomasa aérea}

Para estimar la biomasa aérea (BA) de los árboles se establecieron parcelas circulares de $1000 \mathrm{~m}^{2}$. En cada parcela se evalúo la vegetación arbórea. A cada individuo $(\mathrm{h}>1,30 \mathrm{~m})$ se le midió el diámetro normalizado (DN) y la altura total. Los individuos con una altura total inferior a los $1,30 \mathrm{~m}$ se contabilizaron e identificaron por género. Todos los datos fueron registrados por género y por clase diamétrica, con intervalos de 5 centímetros.

Con los datos recolectados sobre la especie Abies religiosa se elaboró la siguiente ecuación alométrica para las alturas:

$$
\begin{gathered}
\text { Abies religiosa } \\
y=-0.004\left(D N^{2}\right)+0,8427(D N)
\end{gathered}
$$

$$
r^{2}: 0,8427
$$

Para las alturas de la especie Pinus hartwegii se utilizó la ecuación alométrica propuesta por Espinoza (2005):

Pinus hartwegii

$$
y=0,0015\left(D N^{2}\right)+0.3956(D N)
$$

$$
r^{2}: 0,8883
$$




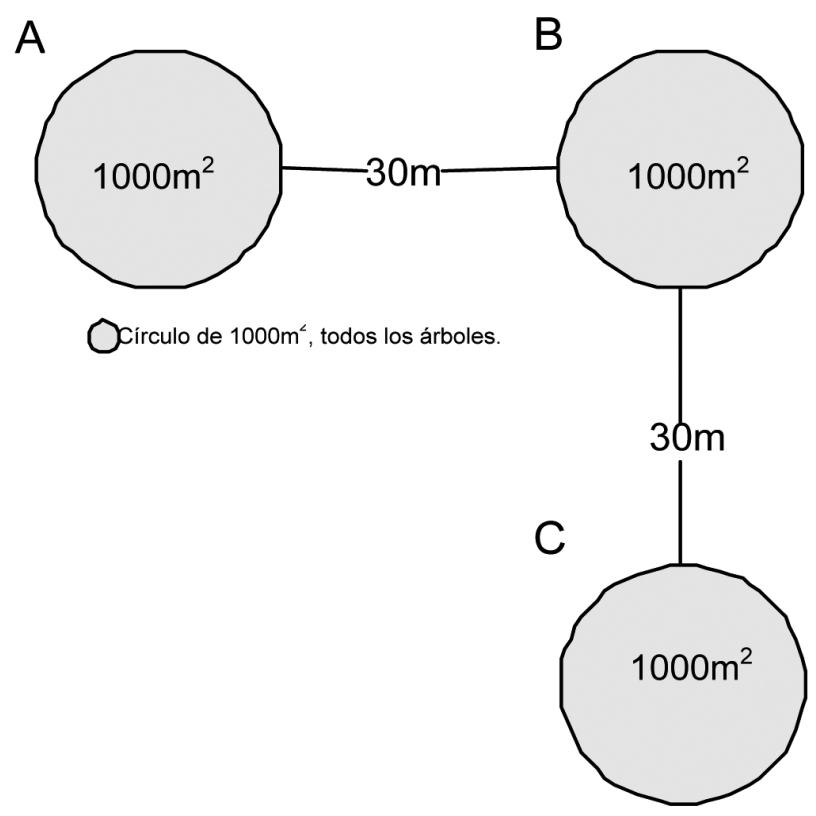

Figura 3. Diseño anidado.

En la estimación de la biomasa aérea para ambas especies se utilizó la siguiente ecuación (Brown y Lugo, 1994):

BA: volumen $x$ densidad de la madera

donde:

Volumen: Área basal x altura

(Grijpma, 2001)

Área basal: $\pi / 4\left(D N^{2}\right)$

(Mostacedo y Fredericksen, 2000)

Para fines de este estudio se tomó el dato de densidad de la madera $\left(\mathrm{t} / \mathrm{m}^{3}\right)$ promedio de las monografías expedidas por el Instituto Nacional de Investigaciones Forestales, Agrícolas y Pecuarias (Musálem y Ramírez, 2003; Musálem y Solís, 2000).
Todos los valores de biomasa se pasaron a carbono mediante un factor de 0,5 MgDM / MGC (IPCC, 1995).

\section{RESULTADOS}

\section{Mapa de vegetación}

Las superficies cubiertas por cada una de las clases de cobertura vegetal y uso de suelo se muestran en el tabla 3 .

El mapa de las diferentes clases de cobertura vegetal y uso de suelo (cvus) se muestra en la figura 4. Para validar cada una de las diferentes cvus se tomaron 266 puntos de verificación bis a bis (usando tres sistemas de posicionamiento global). 


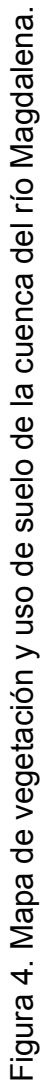
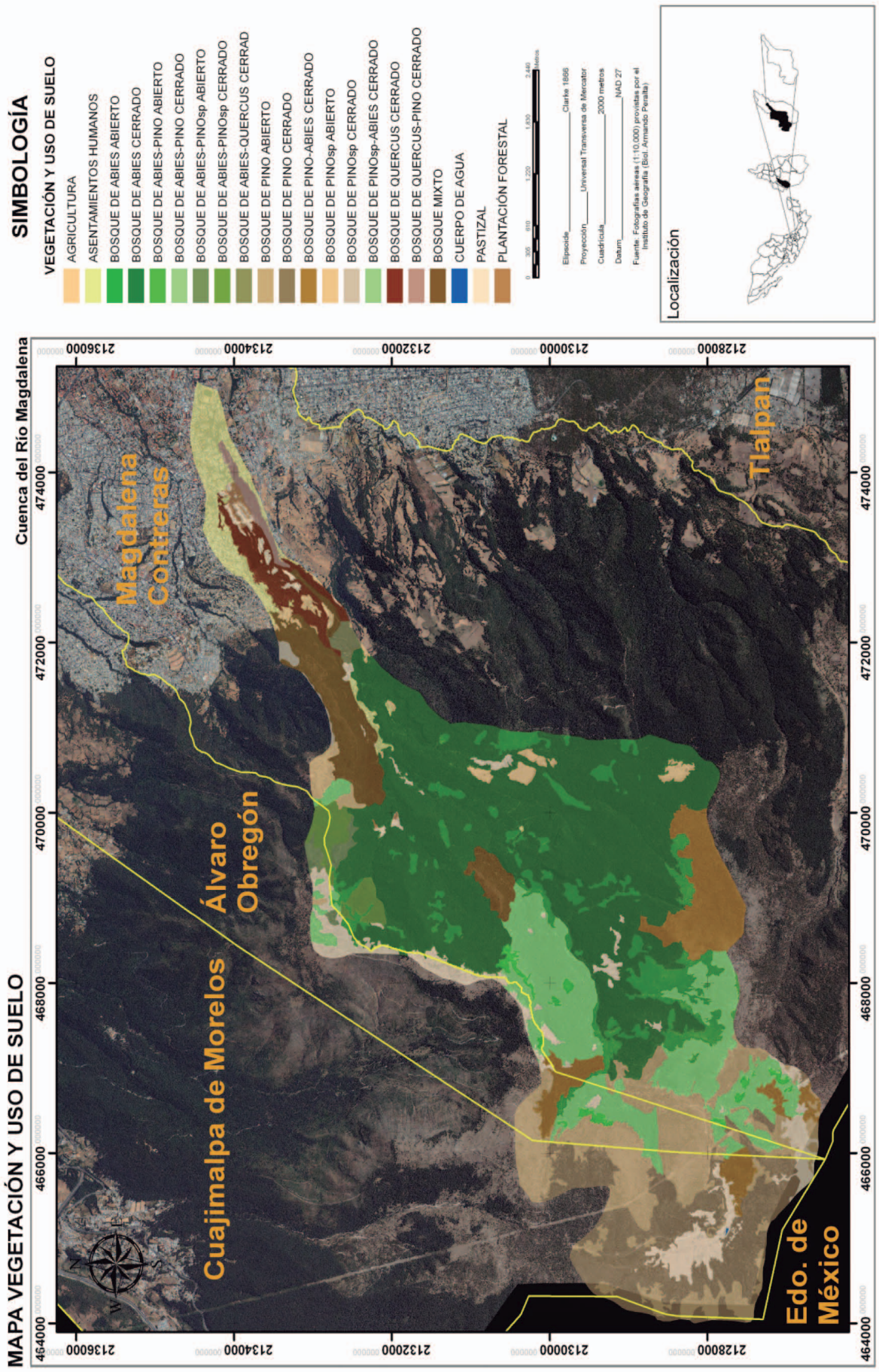
Tabla 3. Superficie que ocupan las diferentes clases de cobertura vegetal y uso de suelo en la CRM.

\begin{tabular}{lrr}
\hline Clases & Área (ha) & $\%$ \\
\hline Agricultura & & 1,07 \\
Asentamientos humanos & 31,98 & 3,34 \\
Bosque de Abies religiosa abierto & 99,60 & 6,14 \\
Bosque de Abies religiosa cerrado & 183,08 & 33,95 \\
Bosque de Abies religiosa-Pinus hartwegii abierto & 1012,09 & 0,66 \\
Bosque de Abies religiosa -Pinus hartwegii cerrado & 19,69 & 12,95 \\
Bosque de Abies religiosa-Pinus sp. abierto & 386,21 & 0,55 \\
Bosque de Abies religiosa-Quercus sp. cerrado & 16,49 & 0,38 \\
Bosque de Quercus sp. cerrado & 11,38 & 2,00 \\
Bosque de Quercus sp.-Pinus sp. cerrado & 59,73 & 0,58 \\
Bosque de Pinus hartwegii abierto & 17,27 & 10,80 \\
Bosque de Pinus hartwegii cerrado & 322,14 & 9,68 \\
Bosque de Pinus hartwegii-Abies religiosa cerrado & 288,56 & 6,45 \\
Bosque de Pinus sp. abierto & 192,19 & 0,69 \\
Bosque de Pinus sp. cerrado & 20,57 & 0,22 \\
Bosque de Pinus sp.-Abies religiosa cerrado & 6,47 & 0,41 \\
Bosque mixto & 12,21 & 4,80 \\
Cuerpos de agua & 143,24 & 0,01 \\
Pastizal & 0,17 & 5,51 \\
Plantación forestal & 164,15 & 0,22 \\
& 6,51 & 100 \\
\hline Total & &
\end{tabular}

\section{Estructura diamétrica del bosque}

Se definieron 11 clases diamétricas para cada uno de los seis tipos de coberturas vegetales muestreadas, a fin de describir la estructura horizontal de los bosques de la cuenca del río Magdalena

Para describir la estructura del bosque, fueron medidos los diámetros de 13,863 árboles y las alturas de 300 de ellos, de los cuales, $61 \%$ fueron Pinus sp., $28 \%$ Abies religiosa, $7 \%$ hojosas, 3\% Cupresus sp. y 1\% Quercus sp. (Fig. 5).

En la figura 5 se observa que la clase diamétrica con más individuos es $<5 \mathrm{~cm}$ $(50 \%)$, teniendo una mayor abundancia de renuevos de Pinus sp. (72\%), seguido de Abies religiosa (22\%) y en menor medida las hojosas (3\%), Cupressus sp. (2\%) y Quercus sp. (1\%). La clase diamétrica con menor número de individuos es $42,6 \mathrm{~cm}-47,5 \mathrm{~cm}(1,8 \%)$ y está compuesta principalmente por Abies religiosa (71\%) y Pinus sp. (23\%) y en menor medida por Cupressus sp. (4,5\%), hojosas $(0,5 \%)$ y Quercus sp. (0,5\%).

Treinta y cinco por ciento de los individuos se encuentra en las clases diamétricas de $5 \mathrm{~cm}$ a 22,5 cm (Fig. 5), donde se presenta una mayor abundancia de Pinus sp. (60\%), seguida de la especie Abies religiosa (21\%), hojosas (14\%) y en menor medida Cupressus sp. (4\%) y Quercus sp. (1\%). 


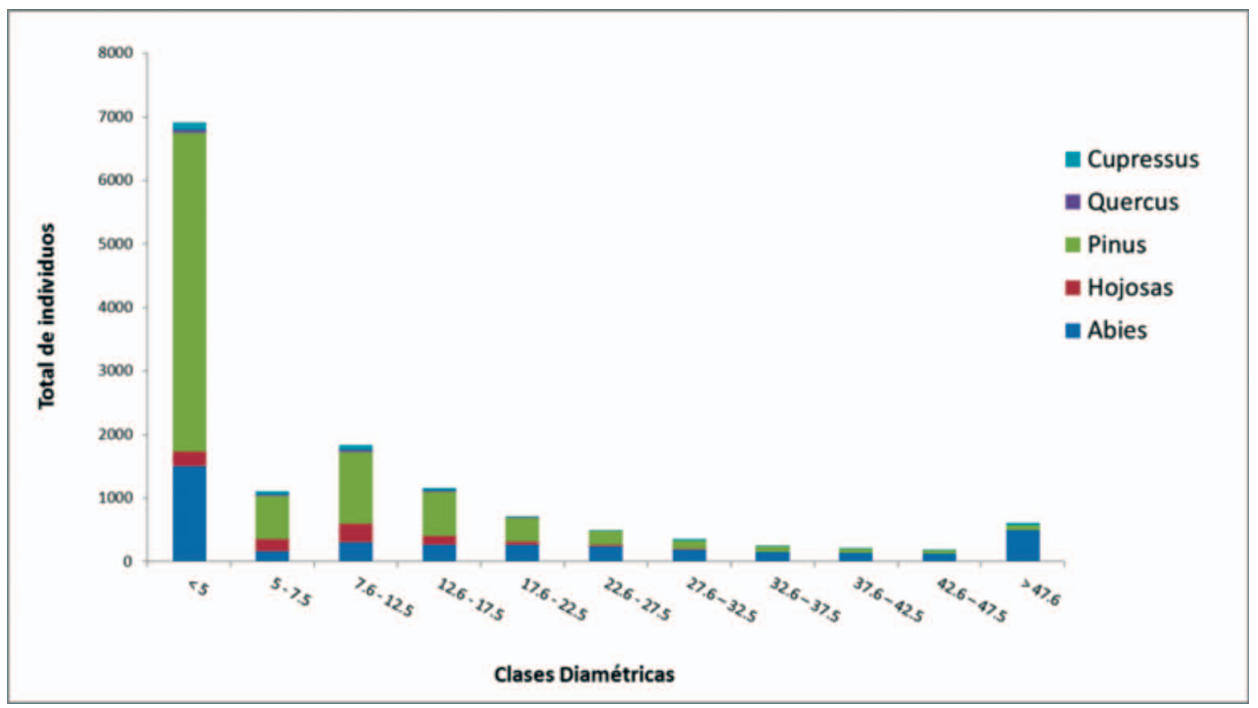

Figura 5. Número de individuos totales, por clase diamétrica, de los bosques de la cuenca del río Magdalena, derivado de 52 sitios de muestreo.

Las clases diamétricas restantes agrupan a $15 \%$ de los individuos. Abies religiosa es la de mayor abundancia con $63 \%$, seguida de Pinus sp. (30\%), Cupressus sp. (4\%), hojosas $(2,5 \%)$ y Quercus sp. $(0,5 \%)$.

\section{Contenido de carbono}

Se determinó el carbono almacenado en cada una de las coberturas vegetales muestreadas (Tabla 4).

Tabla 4. Contenido de carbono por cobertura vegetal.

\begin{tabular}{lcccc}
\hline Cobertura vegetal & Superficie (ha) & $n$ & $\begin{array}{c}\text { Media y EE } \\
\text { (tC/ha) }\end{array}$ & $\begin{array}{c}\text { Intervalo } \\
\text { (tC/ha) }\end{array}$ \\
\hline Bosque de Abies religiosa abierto & 183,08 & 4 & $95,49 \pm 24,51$ & $40,71-155,82$ \\
Bosque de Abies religiosa cerrado & 1012,09 & 22 & $162,91 \pm 16,69$ & $72,37-362,20$ \\
Bosque de Abies - Pinus sp. cerrado & 341,7 & 7 & $106,92 \pm 25$ & $40,30-205,39$ \\
Bosque de Pinus hartwegii abierto & 322,14 & 5 & $21,21 \pm 5,62$ & $6,25-32,29$ \\
Bosque de Pinus hartwegii cerrado & 288,56 & 10 & $47,37 \pm 5,64$ & $18,37-67,84$ \\
Bosque de Pinus sp. - Abies religiosa cerrado & 179,98 & 4 & $39,39 \pm 14,88$ & $27,27-108,73$ \\
& & & &
\end{tabular}

Superficie = área en hectáreas que ocupa la clase vegetal.

$\mathrm{n}=$ número de sitios (cada sitios consta de 3 levantamientos de 0,1 ha).

Media = Contenido de carbono promedio por cobertura vegetal.

$\mathrm{EE}=$ Error estándar.

Intervalo = Valores mínimos y máximos de contenido de carbono por cobertura vegetal. 


\section{DISCUSIÓN}

\section{Estructura diamétrica del bosque}

Los bosques de la Magdalena Contreras son irregulares en cuanto a la edad (en la clasificación forestal corresponde al término multietáneo y homogéneo; Grijpma, 2001), concentrándose la mayor cantidad de individuos en las etapas de regeneración inicial y avanzada $(<10 \mathrm{~cm}$ de DN; Fig. 5).

Se observa que, derivado de las campañas de reforestación, se ha favorecido a diferentes especies del género Pinus, cuya distribución natural no corresponde a la vegetación primaria de los bosques de la Magdalena Contreras. Los Pinus hartwegii son dominantes en la estructura diamétrica $<5 \mathrm{~cm}$ a $22,5 \mathrm{~cm}$ de $\mathrm{DN}$, esto indica que se está desarrollando un proceso de sucesión dirigida y de competencia inducida que afecta a los rodales puros, tanto de Abies religiosa como a los de Pinus hartwegii.

Según Pascual (2003), el Abies religiosa es una especie cuyo crecimiento está en función de la sombra, el suelo y la competencia con otros Abies sp. y especies del sotobosque. Dicha competencia puede provocar la mortandad de las plántulas de Abies religiosa, pues sus raíces son menos capaces que las de las angiospermas (sotobosque) de aprovechar el abastecimiento de agua y nutrimentos en el suelo, en sus etapas iniciales.

Las hojosas representan $7 \%$ del total de la población de las plantas registradas, con dominancia de arbustos en el sotobosque, que parecen ser exógenos y que compiten con las plántulas del estrato arbóreo y que según Nieto (op. cit.), también pueden favorecer el crecimiento de las plántulas de Abies religiosa, al generar un microclima, además, como son estacionales, les provee nutrientes para su crecimiento. Las plantaciones de Cupressus sp. ocupan 3\% del total de la población inmersa en las clases que fueron muestreadas y los Quercus sp. apenas registran 1\%. También se observa la presencia de ruderales que indican perturbación (Acaena elongata).

De acuerdo con los datos registrados, se cree que en un futuro los bosques de la cuenca del río Magdalena cambiarán su dominancia de Abies religiosa a Pinus sp. Posiblemente las condiciones del cambio de clima favorezcan esta transición, ya que de haber un incremento en la temperatura, el género Pinus tendrá mayores probabilidades de sobrevivir, ya que presenta mejor adaptación al ambiente.

\section{Contenido de carbono}

La diferencia de valores de contenido de carbono almacenado, por tipo de cobertura vegetal, presenta variaciones derivadas de:

La edad del arbolado, es decir, los bosques son multietáneos. El crecimiento diferencial derivado de la calidad de sitio refleja diferentes densidades de biomasa arbórea y por ende almacenes de carbono e incrementos disímiles en la misma cobertura vegetal.

El estado de conservación de la masa forestal, la densidad del arbolado y las condiciones actuales de la estructura y composición de cada cobertura vegetal.

La infraestructura de los caminos y las pendientes (accesibilidad).

Otros procesos que afectan a la cuenca y por tanto al contenido de carbono almacenado son: 
Contaminación atmosférica. La ubicación espacial de la cuenca del Río Magdalena es el suroeste de la cuenca de México, dirección a la cual viajan los vientos dominantes, arrastrando la contaminación generada en la ciudad, dando como consecuencia: clorosis, exposición a concentraciones de ozono, afectación de la fitomasa, alteración entre los ciclos reproductivos de diferentes especies, lluvia ácida, retirada de polinizadores y dispersadores, entre otros problemas; esta contaminación también genera condiciones microclimáticas que favorecen el desarrollo y distribución vertical de plagas forestales.

Plagas. La veda forestal en el suelo de conservación del D.F., impide la remoción de individuos que ya están enfermos o que son susceptibles de ser invadidos por organismos patógenos, propiciando el desarrollo de plagas, dando lugar a una disminución en la captura potencial de carbono, la purificación del aire, la generación de oxígeno y un incremento en la distribución de plagas (e.g.: muérdago y descortezador).

Incendios naturales e inducidos. De 1995 a 2004 se han registrado 157 incendios (Flores, 2006) asociados a las sequías y a las actividades humanas; en 1998 se registró uno de los incendios más severos, afectando la copa de los bosques de Abies religiosa, principalmente. Debido a la falta de comunicación de autoridades y de la comunidad Magdalena Atlitic, no se han abierto los canales para darle un saneamiento adecuado a las zonas afectadas; esto también merma de forma directa el potencial de captura de carbono y sus almacenes, así como los continuos de bosque que permiten el desplazamiento de las especies.

\section{Comparación con otros estudios de caso del área de la cuenca del río Magdalena}

Con el fin de conocer los resultados de los estudios relacionados con la estimación del contenido de carbono en el área de la Magdalena Contreras, se realizó un cuadro comparativo (Tabla 5), analizando la superficie de la unidad de muestreo, la superficie total muestreada y el carbono estimado:

Se registraron tres estudios en el caso de Abies religiosa, en los que los autores indican valores que van de las $58 \mathrm{tC} / \mathrm{ha}$ a las $207 \mathrm{tC} / \mathrm{ha}$. Este estudio presenta resultados basados en la densidad del arbolado con valores que van de $95 \mathrm{tC} / \mathrm{ha}$ a $162 \mathrm{tC} / \mathrm{ha}$, lo que permite apreciar diferencias significativas respecto a las sobreestimaciones y subestimaciones en los almacenes de carbono en esta cobertura vegetal, aunado a la intensidad y tipo de muestreo.

Para el bosque de Pinus hartwegii, se comparan los resultados con dos estudios de caso, encontrando valores de carbono almacenado que van de $44 \mathrm{tCl}$ ha a $57,6 \mathrm{tC} / \mathrm{ha}$, en ambos casos no se clasifica esta cobertura vegetal tomando como base la densidad del arbolado y la superficie que ocupa (CORENA, 2001 y Espinoza, 2005).

Este estudio, separa esta clase con base en la densidad del arbolado, observando una diferencia de casi el doble del carbono almacenado entre ambas; es por ello que se sugiere que las interpolaciones y extrapolaciones del carbono almacenado por clase de cobertura vegetal y uso de suelo, se deben hacer con conocimiento de causa. 
Tabla 5. Comparación de los estudios de estimación de contenido de carbono en el suelo de conservación del sur del Distrito Federal y en la cuenca del río Magdalena.

\begin{tabular}{lcccc}
\hline Cobertura vegetal & $\begin{array}{c}\text { Unidad } \\
\text { de muestreo } \\
\text { (ha) }\end{array}$ & $\begin{array}{c}\text { Superficie } \\
\text { (ha) }\end{array}$ & $\begin{array}{c}\text { Carbono } \\
\text { (tC/ha) }\end{array}$ & Fuente \\
& & & \\
\hline
\end{tabular}

Bosque de Abies religiosa

$\begin{array}{lcccc} & 0,1 & 7,4 & 144,07 & \text { CORENA (2001) } \\ & 0,06 & 11,64 & 207,98 & \text { Valenzuela (2001) } \\ \text { Densidad cerrada } & 0,0625 & 1,75 & 58 & \text { Nava (2006) } \\ \text { Densidad abierta } & 0,3 & 6,6 & 162,91 & \text { Este estudio } \\ & 0,3 & 1,2 & 95,49 & \text { Este estudio }\end{array}$

Bosque de Pinus hartwegii

$\begin{array}{lcccc} & 0,1 & 7,4 & 57,61 & \text { CORENA (2001) } \\ \text { Densidad cerrada } & 0,0625 & 1,44 & 44 & \text { Espinoza (2005) } \\ \text { Densidad abierta } & 0,3 & 3 & 47,37 & \text { Este estudio } \\ & 0,3 & 1,5 & 21,21 & \text { Este estudio }\end{array}$

\section{CONCLUSIONES}

En la cuenca del río Magdalena, la cobertura vegetal que registró la mayor cantidad de carbono almacenado en la biomasa aérea fue Abies religiosa, en su densidad cerrada, lo que hace trascendental el diseño de políticas públicas, sobre todo en zonas cercanas a estos tipos de cobertura, dada su relevancia por los servicios ambientales que estos brindan a la Ciudad de México. Además son necesarias campañas de reforestación adecuadas a las condiciones de composición actuales, ya que de seguir las tendencias de dichos programas en la cuenca, se originará una transición en la composición y estructura, principalmente, en los bosques de Abies religiosa, puesto que se registró que la especie más plantada en todas la coberturas vegetales son del genero Pinus, posiblemente derivado de la falta de información técnica-científica, o a su producción en los viveros cercanos.

La variación en las densidades de carbono registradas por tipo de cobertura vegetal, obedecen a múltiples factores (e.g.: calidad de sitio, accesibilidad, cercanía a áreas urbanas, presión de los recursos, presencia de actividades no aptas para este tipo de vocación), pero el principal factor de afectación y permanencia son las actividades antrópicas. Es necesario hacer comparaciones con estudios de caso in situ y ex situ, a fin de enriquecer la posible toma de decisiones sobre el recurso, determinar las técnicas que expliquen mejor el fenómeno, los alcances, las limitaciones y la precisión de los resultados.

El detalle fino del presente estudio permitió disminuir incertidumbres respecto a la distribución de la cobertura vegetal en la cuenca. La escala de análisis del presente estudio permite comparaciones con los mapas temáticos generados por otras instituciones oficiales (e.g.: INEGI, CORENA, Conabio, entre otros), ya que se puede realizar una generalización de las coberturas vegetales para hacerlas compatibles. 


\section{AGRADECIMIENTOS}

Este estudio fue financiado por el Proyecto UNAM:20993-1498-5-XI-07; INE: INE/ A1-055/2007, "Comportamiento y distribución de la plagas de importancia económica en el sector forestal ante un cambio climático en México; financiamiento en parte por Macroproyecto: Manejo de Ecosistemas y Desarrollo Humano, Universidad Nacional Autónoma de México y fondos propios.

\section{REFERENCIAS}

Brown, S. 2001. Measuring carbon in forest: current status and future challenges. Environmental Pollution 116:363-372.

Brown, S. y A. Lugo. 1994. Rehabilitation of tropical lands: A key to sustaining development. Restoration Ecology 2:97-111.

CNA (Comisión Nacional del Agua).1997. Estudio de saneamiento del río Magdalena, Delegación La Magdalena Contreras, Distrito Federal. Gerencia Regional de Aguas del Valle de México. Subgerencia de Calidad del Agua e Impacto Ambiental. Informe final. México. $217 p$.

CORENA (Comisión de Recursos Naturales del Distrito Federal). 2001. Estimación del contenido de carbono en la biomasa en el área del suelo de conservación del Distrito Federal. Octubre. Informe Técnico CORENA. $32 \mathrm{p}$.

Dixon, R.K., S. Brown, R.A. Houghton, A.M. Solomon, M.C. Trexler y J. Wisniewski. 1994. Carbon pools and flux of global forest ecosystems. Science 263:185-190.
Espinoza, M. 2005. Estimación del contenido y captura de carbono en el bosque de Pinus hartwegii de la cuenca alta del río Magdalena, Magdalena Contreras, D.F. Tesis de licenciatura. Facultad de Ciencias. UNAM. México. $72 p$.

Fernández, E.A., F. Uribe, I. Ramírez, B. Apolinar y A. Vázquez. 2002. Evaluación del avance de la mancha urbana sobre el área natural protegida de la Cañada de los Dínamos. Instituto Nacional Ecología. México. Gaceta Ecológica 62:56-67.

Flores, A. 2006. Frecuencia de incendios forestales, su relación con la precipitación y la riqueza de especies vegetales en la cuenca del río Magdalena, D.F., México. Tesis de Licenciatura. Universidad Nacional Autónoma de México. Facultad de Ciencias. México. 53 p.

Galeana, J., 2008, Estimación del contenido y captura potencial de carbono, su valor económico; en la cuenca del río Magdalena, D.F. México. Tesis de Licenciatura. Universidad Nacional Autónoma de México. Facultad de Filosofía y Letras. 95 p.

Grijpma P. 2001. Producción Forestal. 2a. ed. Trillas. México. $134 \mathrm{p}$.

GDF (Gobierno del Distrito Federal). 2000. Programa general del ordenamiento ecológico 2000-2003. Secretaría de Medio Ambiente. Comisión de Recursos Naturales y Desarrollo Rural. Dirección Ejecutiva de Ordenamiento Ecológico. México. 133 p.

Goudie, A., 1990. The Human Impact on the Natural Environmetn. 3a. ed. Basil Blackwell Ltd. Oxford, Reino Unido. 388 p. 
Houghton, R.A. 1991. Tropical deforestation and atmospheric carbon dioxide. Climatic Change 19:99-118.

INEGI (Instituto Nacional Estadística, Geografía e Informática). 2005a. Distrito Federal, Cuaderno estadístico delegacional: Álvaro Obregón. Referencias generales. Aspectos geográficos. México. $16 \mathrm{p}$.

INEGI (Instituto Nacional de Estadística, Geografía e Informática). 2005b. Distrito Federal, Cuaderno estadístico delegacional: Cuajimalpa de Morelos. Referencias generales. Aspectos geográficos. México. $16 \mathrm{p}$.

INEGI (Instituto Nacional Estadística, Geografía e Informática). 2005c. Distrito Federal, Cuaderno estadístico delegacional: Magdalena Contreras. Referencias generales. Aspectos geográficos. México. $16 \mathrm{p}$.

INEGI (Instituto Nacional Estadística, Geografía e Informática). 2005d. Distrito Federal, Cuaderno estadístico delegacional: Tlalpan. Referencias generales. Aspectos geográficos. México. $16 \mathrm{p}$.

IPCC (Intergovermental Panel on Climate Change). 1995. Climate Change 1995. The Suplementary Report to the IPCC Scientific Assessment. Cambridge University Press, Cambridge.

Johns, A. D.,W.V. Reid, D. Simberloff, V.H. Heywood, S.N. Stuart, K.S. Brown Jr. G.G. Brown y R.J. Johns. 1992. Deforestation and species extintion in Tropical Forest. In: Whitmore, T. y J. Sayer (eds.). Tropical deforestation and species extintion. IUCN. Chapman and Hall. Londres, Inglaterra. p:1-14.
Lambin, E.F., 1994. Modelling deforestation processes: a review tropical ecosystem environment observations by satellites, TREES Series B, Research report num. 1. Office of the European Commission, DirectorateGeneral XIII. Luxemburgo. 113 p.

Lund, H. 2006. National forest inventories and global resource assessments. In: Forest and Forest Plants, Encyclopedia of Life Support Systems (EOLSS), EOLSS Publishers, Oxford, Reino Unido. p:29-65.

Masera, O., R.D. Martínez, T. Hernández, A. Guzmán y A. Ordóñez. 2000. Inventario nacional de gases de efecto invernadero 1994-1996. Parte 6: Cambio en el uso del suelo y bosques. Laboratorio de Bioenergía del Departamento de Ecología de los Recursos Naturales del Instituto de Ecología de la UNAM, Campus Morelia-INE, Semarnat, México, D.F, 44 p.

Masera, O. 1995. Carbon mitigation scenarios for mexican forests: methodological considerations and results. Interciencia 20(6):388-395.

Vargas-Mena A., A. y A. Yáñez S. 2004. La captura de carbono en bosques: ¿una herramienta para la gestión ambiental? Gaceta Ecológica 70:5-18.

Mostacedo, B y T. Fredericksen. 2000. Manual de métodos básicos de muestreo y análisis en ecología vegetal. Proyecto de Manejo Forestal Sostenible (BOLFOR) Santa Cruz, Bolivia. $87 \mathrm{p}$.

Murcia, C. 1995. Edge effects in fragmented forest: implications for conservation. Trends in Ecology and Evolution 10(2):58-62. 
Musálem, M.A. y M.A. Solís. 2000. Monografía de Pinus hartwegii. INIFAP, Centro de Investigación Regional del Centro. Secretaría de Agricultura, Ganadería y Desarrollo Rural. 74 p.

Musálem, M.A. y A. Ramírez. 2003. Monografía de Pinus ayacahuite var. veitchii Shaw. INIFAP, Centro de Investigación Regional del Centro. Secretaría de Agricultura, Ganadería y Desarrollo Rural, Pesca y Alimentación. Comisión Nacional Para el Conocimiento y Uso de la Biodiversidad. $364 \mathrm{p}$.

Nava, M. 2005. Carbono almacenado como servicio ecosistémico y criterios de restauración en el bosque de Abies religiosa de la cuenca del río Magdalena, D.F., México. Tesis de Maestría. Universidad Nacional Autónoma de México. Facultad de Ciencias. $70 \mathrm{p}$.

Ordóñez, J.A.B., B.H.J. de Jong, F. García-Oliva, F.L. Aviña, J.V. Pérez, G. Guerrero, R. Martínez y O. Masera. 2008. Carbon content in vegetation, litter, and soil under 10 different landuse and land-cover classes in the Central Highlands of Michoacan, Mexico. Forest Ecology And Management 255(7):2074-2084.

Ordóñez, J.A.B. 1999. Captura de carbono en un bosque templado: el caso de San Juan Nuevo, Michoacán. Tesis de Licenciatura. Universidad Nacional Autónoma de México. Facultad de Ciencias. p:7-9.

Otegui-Acha, M. 2007. Developing and testing a methodology and tools for the inventorying of sacred natural sites of indigenous and tradicional people in Mexico. ALCOA, UICN, Pronatura México A.C., Fundación Rigoberta Menchu Tum. $109 \mathrm{p}$.
IPCC (Panel Intergubernamental de expertos sobre el cambio climático). 2007. Cambio Climático 2007. Informe de síntesis. Resumen para responsables de políticas. Una evaluación del grupo intergubernamental de expertos sobre el cambio climático. $114 \mathrm{p}$.

Pascual, C.N., M.A. Musálem y J. C. Boyás. 2003. Efecto de la vegetación asociada al sotobosque sobre el crecimiento y desarrollo inicial de Abies religiosa (HBK) Schltdl. et Cham. Ciencia Forestal 94(28):7-22.

Rojas-García, F. 2004. Contenido y captura potencial de carbono en el bosque de Pinus hartwegii del Parque Nacional la Malinche: Tlaxcala-Puebla. Tesis de Licenciatura. Universidad Nacional Autónoma de México. Facultad de Ciencias. $68 \mathrm{p}$.

Sathaye, J.A., W.R. Makundi, K. Andrasko, R. Boer, N.H. Ravindranath, P. Sudha, S. Rao, R. Lasco, F. Pulhin, O. Masera, A. Cerón, J.A.B. Ordóñez, $X$. Deying, X. Zhang y S. Zuomin. 2001. Carbon mitigation potential and costs of forestry options in Brazil, China, India, Indonesia, México, Philippines and Tanzania. Mitigation and adaptation Strategies for Global Change 6:185-211.

Schneider, S.H. 1989. The greenhouse effect: science and policy. Science. 243(10):271-281.

Soulé, M. y G. Orians. 2001. Conservation biology: research priorities for the next decade. Island Press. EUA. 127 p.

SFFS. (Subsecretaría Forestal y de Fauna Silvestre). 1994. Inventario Nacional Forestal Periódico 1992-1994. Secretaría de Agricultura y Recursos Hidráulicos (SARH). México. 81 p. 
Valenzuela, H.T. 2001. Estimación de secuestro de carbono en bosques naturales de oyamel en el sur del Distrito Federal. Tesis Profesional. Chapingo, Edo. de México. p:9-60. cuenca del río Magdalena, México. Madera y Bosques 19(1):53-69. 